\title{
Be star disc characteristics near the central object
}

\author{
J. Zorec ${ }^{1}$, M. L. Arias ${ }^{2,3}$, L. Cidale ${ }^{2,3}$, and A. E. Ringuelet ${ }^{2}$ \\ 1 Institut d'Astrophysique de Paris, UMR7095 CNRS, Université Pierre \& Marie Curie, 98bis bd. Arago, 75014 Paris, France \\ e-mail: zorec@iap.fr \\ 2 Facultad de Ciencias Astronómicas y Geofísicas, Universidad Nacional de La Plata, Paseo del Bosque s/n, (1900) La Plata, \\ Argentina \\ 3 Instituto de Astrofísica de La Plata (CONICET-UNLP), Paseo del Bosque s/n, (1900) La Plata, Argentina
}

Received 21 october 2006 / Accepted 13 April 2007

\section{ABSTRACT}

\begin{abstract}
Context. A recent analysis of visual Fe II emission lines of 17 classic Be stars using the self-absorption curve (SAC) method revealed that these lines are optically thick and that they form in circumstellar disc (CD) regions within two stellar radii from the central star on average.

Aims. The aim of this paper is to study the physical characteristics of CD regions situated close to the central star.

Methods. We used the Fe II emission line optical depths derived for a sample of the above mentioned classic Be stars that are seen either nearly pole-on or equator-on. The disc properties sought are then inferred by reproducing the average pole-on and equator-on Fe II line optical depths using simple CD models with different density and temperature distributions.

Results. We found that the CD regions near the star, which account for the average Fe II line opacities obtained with the SAC method, have semi-height scales perpendicular to the equatorial plane $h \gtrsim 0.5 R_{\mathrm{o}}$ and particle density distribution laws $N(R)=N_{\mathrm{o}}\left(R_{\mathrm{o}} / R\right)^{n}$ with $n \lesssim 1$ at $R \lessgtr 3 R_{\mathrm{o}}\left(R_{\mathrm{o}}\right.$ is the stellar radius; $N_{\mathrm{o}}$ is the particle density at $\left.R=R_{\mathrm{o}}\right)$. Multi-scattering Monte Carlo simulations show that CD with particle density distributions $N \sim R^{-n}$, where $n$ depends on the distance $R$ and $n \sim 0.5$ near the star, might account for the near-UV spectroplorarimetry of Be stars. CD with enhanced scale heights could explain the $[\mathrm{H} \alpha, E(J-L)]$ correlation as they may produce about the same $\mathrm{H} \alpha$ line emission, but larger IR flux excesses than thin discs. The enlarged CD scale heights do not contradict the existing interferometric measurements and should enable us to treat more consistently Balmer line emission formation in Be stars.
\end{abstract}

Key words. stars: emission-line, Be - stars: circumstellar matter - line: formation - polarization

\section{Introduction}

Arias et al. (2006) analyzed Fe II emission lines in the visual spectrum of 17 southern classical Be stars by applying the selfabsorption curve (SAC) method (Friedjung \& Muratorio 1987; Muratorio \& Friedjung 1988). The self-absorption curve is related to the line-of-sight photon escape probability associated with the model of the line formation region (Kastner 1999) and provides information on the line optical depth regime. This method gives the average optical depth of emission lines that belong to a given reference line-multiplet, $\tau_{\mathrm{o}}$, and yields an estimate of the extent of the line emitting region. Arias et al. (2006) concluded that the studied Fe II lines are optically thick, having an average opacity of $\overline{\tau_{\mathrm{o}}}=2.4 \pm 0.9$ in the central wavelength of multiplet 38 (hereafter $m 38$ ), and that in most cases they form between 1 and 3 stellar radii. Thus, in this paper we use Fe II line optical depths to infer some insights into the density and temperature distributions in CD that are close to the central star and discuss the incidence they can have on the polarimetric, photometric, interferometric and spectroscopic data.

\section{Circumstellar disc characteristics}

\section{1. "Probe" star+circumstellar disc}

The goal of the present contribution is to explore the possible physical characteristics of the CD layers where Fe II emission lines are formed. For this purpose, we shall use a unique or average "probe" star $+\mathrm{CD}$ system. Thus, we assume that the optical depths of the probe CD seen pole-on and equator-on can be defined by averaging those obtained in Arias et al. (2006). Knowing that Fe II level populations are dependent on the stellar radiation field and that the opacities used in the calculation of averages should correspond to gaseous environments with similar excitation conditions, we gathered those objects studied in Arias et al. (2006) that have similar effective temperatures (see Table 1). The "probe" central star is then defined by averaging the fundamental parameters of the selected objects. This gives: $\overline{T_{\text {eff }}}=21000 \mathrm{~K}, \bar{M}=9.5 M_{\odot}, \overline{R_{\mathrm{o}}}=6.7 R_{\odot} ; \overline{V_{\mathrm{c}}}=456 \mathrm{~km} \mathrm{~s}^{-1}$ (critical rotational velocity), which are parameters that closely correspond to a B2 type object in the second half of the main sequence evolutionary phase, for which the highest frequency of Be stars is found (Zorec \& Briot 1997; Zorec et al. 2005).

The effective temperature, mass, radius and inclination angle $i_{*}$ of stars given in Table 1 were obtained with model atmospheres and evolutionary tracks of fast rotating stars (Frémat et al. 2005; Zorec et al. 2005). This table also shows the mean optical depth $\tau_{\mathrm{o}}$ of the respective Fe II lines of $m 38$ and the radii of their formation region derived with the SAC method.

We characterize the "probe" CD with average opacities determined with the SAC method of discs observed in two extreme directions, i.e. pole-on and equator-on. In Table 1 there are two genuine pole-on Be stars ( $\operatorname{stars} n_{\mathrm{o}}^{*}=5$ and 11 with inclination angles $i_{*} \lesssim 20^{\circ}$ ) from which we obtain $\overline{\tau_{\mathrm{po}}}=2.0 \pm 0.2$ and $\overline{T_{\text {eff }}} \simeq 20900 \mathrm{~K}$. We can then attempt a similar estimate of the average pole-on opacity with stars $n_{\mathrm{o}}^{*}=3,5,7,9,10$ and 11 , using the approximation $\tau_{\mathrm{po}} \simeq \tau_{\mathrm{o}} \times \cos i_{*}$, to obtain $\overline{\tau_{\mathrm{po}}}=1.9 \pm 0.9$. 
Table 1. Be stars and the Fe II emission line formation parameters in the respective circumstellar discs.

\begin{tabular}{rrcrrccc}
\hline \hline Object & $n_{\mathrm{o}}^{*}$ & $T_{\text {eff }}$ & $M / M_{\odot}$ & $R_{\mathrm{o}} / R_{\odot}$ & $i_{*}[\mathrm{deg}]$ & $\tau_{\mathrm{o}}$ & $R / R_{\mathrm{o}}$ \\
\hline HD 41335 & 1 & 20900 & 10.1 & 5.8 & 69 & 2.0 & 2.0 \\
HD 45725 & 2 & 17800 & 7.0 & 5.3 & 67 & 3.1 & 4.2 \\
HD 48917 & 3 & 20300 & 10.5 & 10.0 & 45 & 2.0 & 2.3 \\
HD 50013 & 4 & 24600 & 11.4 & 5.5 & 37 & 1.7 & 1.1 \\
HD 56139 & 5 & 19500 & 6.3 & 5.5 & 17 & 2.2 & 1.7 \\
HD 58978 & 6 & 24400 & 10.6 & 4.2 & 55 & 2.9 & 1.3 \\
HD 88661 & 7 & 21500 & 8.3 & 4.4 & 39 & 4.5 & 1.5 \\
HD 91465 & 8 & 17800 & 7.7 & 7.7 & 67 & 2.2 & 2.8 \\
HD 105435 & 9 & 22300 & 9.2 & 5.2 & 42 & 1.2 & 2.3 \\
HD 112091 & 10 & 20400 & 7.9 & 3.9 & 36 & 2.2 & 3.0 \\
HD 120991 & 11 & 22200 & 9.8 & 7.2 & 13 & 1.8 & 1.8 \\
HD 124367 & 12 & 17500 & 5.7 & 4.1 & 63 & 2.9 & 3.0 \\
HD 157042 & 13 & 21800 & 9.0 & 4.9 & 53 & 1.8 & 1.7 \\
HD 158427 & 14 & 18000 & 6.2 & 4.4 & 51 & 1.7 & 1.5 \\
\hline
\end{tabular}

$\tau_{\mathrm{o}}=$ optical depth in the central wavelength of $m 38 ; R / R_{\mathrm{o}}=$ radius of the Fe II line formation region.

This implies that we can assume $\tau_{\mathrm{po}}=2.0$ for our "probe" CD. Concerning $\overline{\tau_{\mathrm{eq}}}$, we see that in Table 1 there is only one genuine equator-on $\operatorname{star}\left(i_{*} \gtrsim 60^{\circ}\right)$ having the required effective temperature and $\tau_{\text {eq }}=2.0\left(n_{\mathrm{o}}^{*}=1\right)$. Other equator-on stars, like $n_{\mathrm{o}}^{*}=2$, 8 and 12 lead to $\overline{\tau_{\mathrm{eq}}}=2.5 \pm 0.9$, but their effective temperatures are a little low $\left(\overline{T_{\text {eff }}} \simeq 17500\right)$ to define with them the "probe" CD. Hence, for stars having intermediate inclinations $i_{*}$ and $T_{\text {eff }} \sim 21000 \mathrm{~K}$, the only thing we can do is to assume that probably $\overline{\tau_{\text {eq }}} \gtrsim \overline{\tau_{\mathrm{o}}}$, as we cannot infer their actual optical depths using $\tau_{\mathrm{eq}} \sim \tau_{\mathrm{o}} \cos i_{*} / \cos (i \rightarrow \pi / 2)$. Therefore, we shall adopt a parameterized optical depth of the "probe" CD seen equator-on using the following plausible values: $\overline{\tau_{\mathrm{eq}}}=2.0,4.0,6.0$.

In order to characterize roughly the structure of the studied CD layers, we assume that they are flat, i.e. semi-height $h / R_{\mathrm{o}}=$ constant $\left(R_{\mathrm{O}}=\right.$ stellar radius) and have a particle distribution given by $N(R)=N_{\mathrm{o}}\left(R_{\mathrm{o}} / R\right)^{n}$ in the equatorial plane, but it is uniform perpendicularly to the equator. In this section we assume that the power index $n$ is constant over the entire studied line formation region. We note, however, that this CD model is applicable only to the regions where Fe II lines are formed, and that it might not be viable for the outer $\mathrm{CD}$ regions. Three temperature distributions are also tested: i) radiative-equilibrium (RE): this temperature distribution is similar to that obtained by Carciofi \& Bjorkman (2006); ii) discs entirely isothermal with $T=T_{\mathrm{Ba}}$, where $T_{\mathrm{Ba}}=9 \times T_{\text {eff }}^{0.73} \simeq 12900 \mathrm{~K}$ designates the temperature due to heating provided by the ionizing stellar Balmer photons (Moujtahid et al. 1999); iii) non-radiative equilibrium (non-RE), which is represented by the chromospheric-like structure used by Cidale \& Ringuelet (1993), Cidale \& Vázquez (1995) and Cidale (1998), assuming a maximum temperature of $30000 \mathrm{~K}$ followed by an isothermal region with $T=T_{\mathrm{Ba}}$ (see Sect. 3.1). These temperature distributions are shown in Fig. 1a.

\subsection{Line source function}

In this section we briefly discuss the behavior of the line source function and level populations of the studied Fe II lines for the different temperature and density structures mentioned above.

We computed non-LTE departure coefficients $b_{1}$ and $b_{\mathrm{u}}$ of the average $m 38$ transition $\left(b_{\mathrm{u}, 1}=N_{\mathrm{u}, 1} / N_{\mathrm{u}, 1}^{*}\right.$, where $N_{\mathrm{u}, 1}^{*}$ designates the LTE upper/lower level populations) in a spherically symmetric extended atmosphere using the equivalent-two-levelatom approach. We used the statistical equilibrium equations to determine the population ratio that enters the line source function. The radiative transfer equation is solved by means of a Feautrier's elimination scheme, for each line and for the continuum (Cidale 1998). The calculated radiation fields are then used to improve the transition rates. This procedure is iterated until the convergence of occupation numbers is reached. We assume an Fe II ion model that explicitly includes 42 bound energy terms and a continuum. Fe III is represented as a one-level atom. The photo-ionization cross sections and oscillator strengths are from Nahar (1995) and the collision cross sections from Zhang $\&$ Pradhan (1995). Figure 1b shows the non-LTE departure coefficients $b_{1}$ and $b_{\mathrm{u}}$ for the average transition of $m 38$ calculated for different temperature distributions and a particle density distribution law $N(R)=N_{\mathrm{o}}\left(R_{\mathrm{o}} / R\right)^{n}$ with $N_{\mathrm{o}}=10^{13} \mathrm{~cm}^{-3}$ and $n=2$. Calculations show that $b_{1}$ and $b_{\mathrm{u}}$ depend individually very little on the power $n$ of the density law, but that they are sensitive to the temperature. However, this temperature dependency cancels almost entirely in the $b_{\mathrm{u}} / b_{1}$ ratio, which becomes then a strong function of the diluted stellar radiation. As a consequence, the source function $S_{1}$ of $m 38$, calculated for all the adopted temperature structures and normalized to their value at the stellar surface, differ very little from each other and decreases rapidly with the distance $R$ from the star (see Fig. 1c).

Then, introducing the calculated source functions in $I(x, y)=$ $\int_{z} S_{1} \mathrm{e}^{-\tau_{1}} \mathrm{~d} \tau_{1}$, it can be proved that more than $95 \%$ of the contribution to the radial specific intensity at the $\mathrm{CD}$ equator comes from regions lying within less than some 3 stellar radii from the central star. This confirms the result obtained with the SAC method that the Fe II line emission formation regions lie near the central star.

\subsection{Inferred $C D$ density distribution}

Using the mean opacities $\overline{\tau_{\mathrm{eq}}}$ and $\overline{\tau_{\mathrm{po}}}$, we can attempt to estimate the typical $n$-index of the density distributions and the corresponding electron density $N_{\mathrm{e}}^{\mathrm{o}}$ of the $\mathrm{CD}$ at $R=R_{\mathrm{0}}$. In order to do so, we represent the optical depth of Fe II lines obtained by the SAC method as follows:

$\sigma \overline{S_{1}}\left(1-\mathrm{e}^{-\overline{\tau_{\mathrm{o}}}}\right)=\iint_{\sigma} \mathrm{d} x \mathrm{~d} y \int_{z} S_{1}(x, y, z) \mathrm{e}^{-\tau_{1}} \kappa(x, y, z) \mathrm{d} z$

where $S_{1}$ is the Fe II line source function; $\sigma$ is the area of the effective emitting CD surface, seen either equator-on or pole-on; $\kappa$ is the line absorption coefficient, and $(x, z)$ are coordinates in the sky plane, perpendicular to the $y$ coordinate directed towards the observer. $\overline{S_{1}}$ is the optical depth-weighted mean of the source function, averaged over the apparent area $\sigma$. The integration in (1) is not carried out over a previously limited region, but over the whole disc extent where the product $S_{1} \mathrm{e}^{-\tau} K$ is not negligible.

The optical depth in the Fe II line center is given by (Jefferies 1968):

$\tau_{1}=1.5 \times 10^{-15}\left[\lambda_{\mathrm{o}} f / v_{\mathrm{D}}\right] \int_{y} N_{\mathrm{l}}(x, y, z) \mathrm{d} y$,

where $\lambda_{\mathrm{o}}$ is given in $\AA$; $f$ is the oscillator strength; $v_{\mathrm{D}}$ is the Doppler width of the line in $\mathrm{km} \mathrm{s}^{-1}$ and $N_{1}$ is the number density of the absorbing level. As noted in Sect. 2.2, the level populations and the source function $S_{1}$ of $m 38$ are calculated using the non-LTE approximation and the solar number-abundance ratio $N(\mathrm{Fe}) / N(\mathrm{H})=3.2 \times 10^{-5}$. 

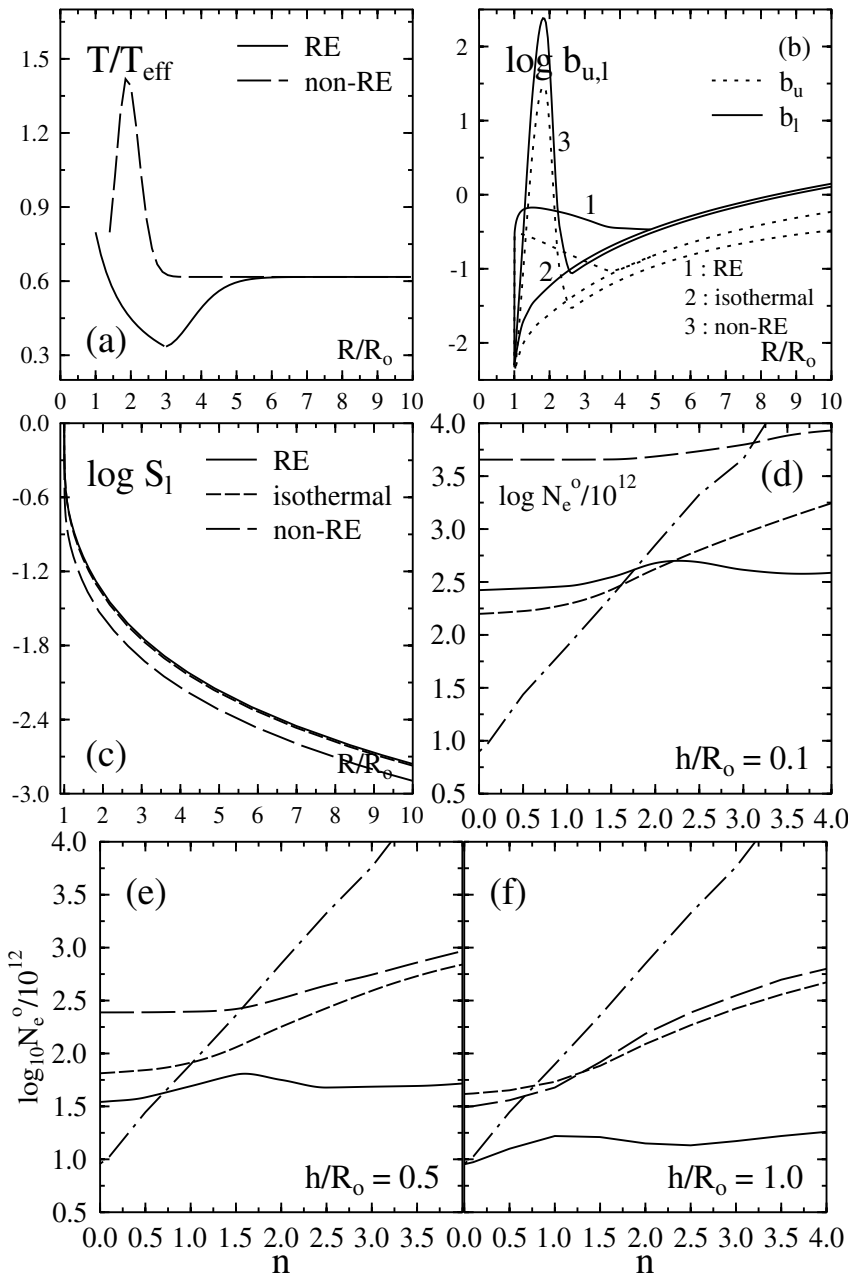

Fig. 1. a) RE and non-RE temperature distributions used in the calculations; the isothermal distribution is for $T=T_{\mathrm{Ba}}$; b) non-LTE deviation coefficients of the average $m 38$ upper and low level obtained for $n=2$ and $\left.N_{\mathrm{e}}^{\mathrm{o}}=10^{13} \mathrm{~cm}^{-3} ; \mathbf{c}\right)$ normalized source functions of the average $m 38$ transition obtained for the same conditions as in b); d) to f) $N_{\mathrm{e}}^{\mathrm{o}}$ densities against the power $n$ of radial density distributions in discs with different temperature and constant semi-height $h / R_{\mathrm{o}}$ that reproduce the observed average opacities $\overline{\tau_{\mathrm{po}}}=2.0$ and $\overline{\tau_{\mathrm{eq}}}=4.0$. The intersection of equatoron (dash-dot lines) with pole-on solutions determines the values of $N_{\mathrm{e}}^{\mathrm{o}}$ and $n$ of $\mathrm{CD}$ regions where the Fe II lines can be formed. All curves from (d) to (f) refer to the same temperature structure as indicated in c) solid line $=$ RE, short-dashed $=$ isothermal, and long-dashed $=$ non-RE

For a given $\mathrm{CD}$, we have calculated the physical quantities needed to account for the average optical depths when this same disc is seen in the pole-on and equator-on directions. The integrations done in Eqs. (1) and (2) take into account all apparent differences of the disc structure seen according to these two orientations. However, at each projection, the integrated quantities issued from (1) and (2) depend on the adopted disc parameters $\left(N_{\mathrm{e}}^{\mathrm{o}}, n, H / R_{\mathrm{o}}\right)$, whatever the region in the disc that contribute the most to the emitted radiation in that direction and whatever the corresponding optical depths. Then, according to the adopted $\mathrm{CD}$ density and temperature structure, our results refer directly to the adopted disc parameters $\left(N_{\mathrm{e}}^{\mathrm{o}}, n, H / R_{\mathrm{o}}\right)$. Two independent series of $N_{\mathrm{e}}^{\mathrm{o}}$ and $n$ values that account for $\overline{\tau_{\mathrm{po}}}=2.0$ and the given $\overline{\tau_{\text {eq }}}$ are obtained for each adopted CD temperature structure. The intersection of these series identifies the set of $\left(N_{\mathrm{e}}^{\mathrm{o}}, n, H / R_{\mathrm{o}}\right)$ parameters which are meant to represent the region where the Fe II lines are formed. The $\left(N_{\mathrm{e}}^{\mathrm{o}}, n, H / R_{\mathrm{o}}\right)$ solutions thus obtained are
Table 2. Solutions for $\log N_{\mathrm{e}}^{\mathrm{o}}$ and the power $n$ of radial density distributions in discs with constant semi-height $h / R_{\mathrm{o}}$ and different temperature structures for $\overline{\tau_{\mathrm{po}}}=2.0$ and several values of $\overline{\tau_{\mathrm{eq}}}$.

\begin{tabular}{ccccccc}
\hline \hline \multicolumn{8}{c}{ RE } & \multicolumn{3}{c}{ isothermal } & \multicolumn{2}{c}{ non-RE } \\
$h / R_{\mathrm{o}}$ & $n$ & $\log \frac{N_{\mathrm{e}}^{\mathrm{o}}}{10^{12}}$ & $n$ & $\log \frac{N_{\mathrm{e}}^{\mathrm{o}}}{10^{12}}$ & $n$ & $\log \frac{N_{\mathrm{e}}^{\mathrm{o}}}{10^{12}}$ \\
\hline \multicolumn{7}{c}{$\overline{\tau_{\mathrm{eq}}}=2.0$} \\
\hline 0.1 & 2.11 & 2.63 & 1.97 & 2.49 & 3.53 & 3.83 \\
0.5 & 1.13 & 1.73 & 1.42 & 2.04 & 2.01 & 2.52 \\
1.0 & 0.47 & 1.09 & 1.04 & 1.74 & 0.93 & 1.66 \\
1.5 & 0.07 & 0.74 & 0.84 & 1.58 & 0.62 & 1.42 \\
\hline \multicolumn{7}{c}{$\overline{\tau_{\mathrm{eq}}}=4.0$} \\
\hline 0.1 & 1.76 & 2.61 & 1.60 & 2.47 & 3.07 & 3.81 \\
0.5 & 0.70 & 1.63 & 0.98 & 1.91 & 1.55 & 2.43 \\
1.0 & 0.00 & 0.95 & 0.71 & 1.69 & 0.55 & 1.57 \\
1.5 & -0.43 & 0.64 & 0.51 & 1.52 & 0.28 & 1.34 \\
\hline \multicolumn{7}{c}{$\overline{\tau_{\mathrm{eq}}}=6.0$} \\
\hline 0.1 & 1.47 & 2.54 & 1.29 & 2.37 & 2.78 & 3.77 \\
0.5 & 0.49 & 1.58 & 0.78 & 1.88 & 1.32 & 2.41 \\
1.0 & -0.25 & 0.90 & 0.53 & 1.66 & 0.38 & 1.54 \\
1.5 & -0.60 & 0.60 & 0.35 & 1.50 & 0.13 & 1.32 \\
\hline
\end{tabular}

given in Table 2. Figures $1 \mathrm{~d}$ to $\mathrm{f}$ show the curves obtained for $\overline{\tau_{\mathrm{eq}}}=4.0$ and $\overline{\tau_{\mathrm{po}}}=2.0$. These curves are identified as in Fig. 1c: solid line $=\mathrm{RE}$, short-dashed line $=$ isothermal, and long-dashed line $=$ non-RE. Since the curves related to $\overline{\tau_{\mathrm{eq}}}$ (dash-dot lines) and for the three tested temperature distributions are very close to each other, we plot only those for CD in RE. The equator-on curves apparently do not depend on the temperature structure, because Fe II lines are optically thick and a strong contribution to the emergent $I(x, y)$ comes from the layers where all adopted temperature laws converge to the same temperature $T=T_{\mathrm{Ba}}$. On the contrary, the dependence with temperature of pole-on curves does not rely on the line opacity regime. In fact, the emergent ray intensity $I(R)$ entering the observed flux $2 \pi \int I(R) R \mathrm{~d} R$ is a function of a given $T(R)$ law, which at any distance $R$ from the star is almost constant over the integration range in the $z$-coordinate. We note that solutions with $N_{\mathrm{e}}^{\mathrm{o}}>10^{14} \mathrm{~cm}^{-3}$ are probably unrealistic, as these densities are similar, or larger than those in photospheric layers where the $\mathrm{H} \alpha$ absorption line is formed. It is also expected that for such high CD densities the second component of the Balmer discontinuity (BD), in emission or in absorption, would strongly overlap the photospheric one (Rohrmann et al. 2003), which is not observed. We consider then that acceptable solutions for the Fe II line emitting regions should have $N_{\mathrm{e}}^{\mathrm{o}} \lesssim 10^{14} \mathrm{~cm}^{-3}$. This result rules out CDs with the thinnest scale heights in radii $R \lesssim 3 R_{\mathrm{o}}$, including the simple isothermal Keplerian CD. This corresponds roughly to all solutions marked in italics in Table 2. The "acceptable" solutions are not unique, but for any adopted temperature distribution, they have a somewhat enhanced scale height near the star and particle density distributions with $n \lesssim 1$. For some solutions, where $h / R_{\mathrm{o}} \gtrsim 1$, we see that $n \lesssim 0$ which corresponds to a density distribution that increases outwards in regions close to the star.

From the results obtained in this section (see Table 2) we conclude that $\mathrm{CD}$ in Be stars should have particle density distribution laws $N(R) \sim R^{-n}$ with $n \lesssim 1$ for $1 \lesssim R / R_{\mathrm{O}} \lesssim 3$. On the other hand, the analysis carried on the far-IR energy distributions by Waters et al. $(1987,1991)$ have shown that $2 \lesssim n \lesssim 3.5$ at distances $4 \lesssim R / R_{\mathrm{o}} \lesssim 8$ (see Sect. 4.2 for the radii of IR radiation formation regions). This means that the power index $n$ of the density distribution must be a function of $R$. Similarly, 
Carciofi et al. (2006) have found that the $J H K L$ photometry and visual to near-IR spectropolarimetry of $\delta$ Sco during its active phase (years 2001-2004) can be modeled using a CD in RE having a radial density distribution $\rho(R) \sim R^{n}$, where $n$ is a function of $R$, and in particular, at radii $R \lesssim 3 R_{\mathrm{o}}$ is flatter than in an isothermal, viscosity governed disc, where $\rho \propto R^{-3.5}$. For our numerical simulations in Sects. 4.1 and 4.2 we adopt $n=n(R)=$ $0.5+2.56 \mathrm{e}^{-23 /\left[\left(R / R_{\mathrm{o}}\right)-1\right]^{5}}$ which ensures that $n$ changes smoothly from $n=0.5$ to $n=3.0$ at $R \sim 3 R_{\mathrm{o}}$ (see Fig. 3a).

Finally, we note that the high similarity of the pole-on and more or less equator-on optical depths obtained with the SAC method can be due to the short equatorial region over which the $\mathrm{Fe}$ II lines are formed, and to the enhanced scale heights of discs near the star. In discs, which are thin over most of their extent, we would expect that $\overline{\tau_{\mathrm{eq}}} \gg \overline{\tau_{\mathrm{o}}}$.

\section{On the disc thickness near the central star}

In Sect. 2 we have concluded that CDs might have scale heights $h / R_{\mathrm{o}} \gtrsim 0.5$ near the star and a power particle distribution law with $n \lesssim 1$ which is not of current use for Be stars. Now, we briefly comment on some possible physical circumstances that may lead to CD scale heights close to the star larger than those in isothermal Keplerian discs.

\subsection{The thin-disc approximation}

In a cylindrical system of coordinates $(R, \phi, z)$ ( $z$ is perpendicular to the equatorial plane), the $R$ and $z$-components of a steady state momentum conservation condition for an axially symmetric $C D$ in hydrostatic equilibrium in the $z$-direction $\left(v_{z}=0\right)$ are (Frank et al. 1992):

$$
\begin{aligned}
& \frac{1}{\rho} \frac{\partial P}{\partial R}=-\frac{G M}{R^{2}}+\frac{v_{\phi}^{2}}{R}-v_{\mathrm{R}} \frac{\partial v_{\mathrm{R}}}{\partial R} \\
& \frac{1}{\rho} \frac{\partial P}{\partial z}=\frac{\partial}{\partial z}\left[\frac{G M}{\left(R^{2}+z^{2}\right)^{1 / 2}}\right],
\end{aligned}
$$

where $P$ is the sum of gas and radiation pressures, $\rho$ is the gas density, $M$ is the stellar mass, $v_{\phi}=R \Omega(R)$ is the circular velocity $\left(\Omega(R)\right.$ is the angular velocity in the disc) and $v_{\mathrm{R}}$ is the velocity in the radial direction. According to the assumed velocity field and physical conditions in the $\mathrm{CD}$, different types of solutions for the density distribution can be drawn from (3) (cf. Schmitz 1983; Frank et al. 1992; Rohrmann 1997). For simplicity, in this paper we assume that $\rho(R, z)=\rho_{\mathrm{R}}(R) \times \rho_{z}(z)$ and a temperature structure $T=T(R)$, which avoids treating the $R$-component of Eqs. (3). If $z \ll R$, from the second equation in (3) it follows that:

$\rho(R, z)=\rho_{\mathrm{R}}(R) \exp \left\{-\left[z / \sqrt{2} H_{z}(R)\right]^{2}\right\}$,

where $H_{z}(R)=\left(v_{\mathrm{s}} / v_{\phi}\right) R$ is the disc scale height in the $z$-direction, $v_{\mathrm{s}}=(P / \rho)^{1 / 2}$ is the sound speed. It is currently assumed that $\rho_{\mathrm{R}}(R) \approx\left(R_{\mathrm{o}} / R\right)^{n}$ with $n \gtrsim 2$, which relies on studies of far-IR and sub-mm energy distributions (Waters et al. 1987, 1991). This radiation originates at distances $R \gtrsim 4 R_{\mathrm{o}}$ from the central star (see Sect. 4.2), which means that $n \gtrsim 2$ may not be appropriate for the CD layers close to the star. Making $z \approx H_{z}$, the thin disks condition $z \ll R$ can be rewritten as $v_{\mathrm{s}} \ll v_{\phi}$, useful to discuss the thickness of CDs. The parameters of the probe star, together with $v_{\phi}=v_{\mathrm{K}}(R)=(G M / R)^{1 / 2}$ for Keplerian rotation, give $v_{\mathrm{s}} \lesssim 10 \mathrm{~km} \mathrm{~s}^{-1}$ and $v_{\mathrm{K}} \sim 320 \mathrm{~km} \mathrm{~s}^{-1}$ at $R / R_{\mathrm{o}}=2$, which implies that close to the star it is $v_{\mathrm{s}} \ll v_{\mathrm{K}}$. Although this might justify the use of the thin disc approximation, considering that: 1) the central star is an active object, and/or 2) there may be magnetic fields, the condition $v_{\mathrm{s}} \ll v_{\phi}$ may not longer hold near the star. Let us discuss this briefly:

1) The active character of Be stars and hence that near the star there can be temperatures as high as $T_{\text {eff }} \ll T_{\mathrm{e}} \lesssim 10^{5} \mathrm{~K}$ (Lamers \& Snow 1978; Fontenla et al. 1981) is revealed by the presence of super-ionized transitions in the far-UV (N V, C IV, $\mathrm{Si}$ IV, etc.), and by lines of $\mathrm{Al}$ III, which are present in all $\mathrm{Be}$ sub-spectral types and $V \sin i$ (Ringuelet \& Torres, private communication). For such temperatures, the radiation pressure is a non-negligible force term in relations (3). Then, following Frank et al. (1992, p. 76) we calculate $v_{\mathrm{s}}=(P / \rho)^{1 / 2}$ using for $P$ the sum of the gas and radiation pressures $P=P_{\text {gas }}+P_{\text {rad }}$ (we approximate $P_{\text {rad }} \propto T^{4}$ ). It follows then that CD regions with temperatures $T_{\mathrm{e}} \gtrsim 3 \times 10^{4}$ have $v_{\mathrm{s}} \gtrsim v_{\mathrm{K}} / 3$, which can invalidate the condition $z \ll R$ and makes Eq. (3) produce geometrically thick discs (see Rohrmann 1997).

Concerning the distribution $\rho_{\mathrm{R}}(R)$, we note that the mass-loss phenomenon in Be stars has two known forms: 1) continuous and variable winds with average rates $\dot{M} \sim 10^{-9} M_{\odot} / \mathrm{yr}$ (Snow 1982); 2) discrete mass ejections of $\Delta M \lesssim 10^{-10} M_{\odot}$ (Guinan \& Hayes 1984; Brown \& Wood 1992; Hanuschik et al. 1993; Floquet et al. 2000; Hubert et al. 2000; Zorec et al. 2000). Even though the origin of the discrete mass ejections is still unknown, the causes suggested sometimes are based on non-radial pulsations (Rivinius et al. 1998) and on instabilities associated with magnetic fields $B \sim 10^{2} \mathrm{G}$ in fast rotating B-type stars (Apparao et al. 1987). The interaction of winds with the circumstellar clumps produced by the discrete mass ejections can build radial density structures $\rho_{\mathrm{R}}(R) \propto R^{4}$ up to some critical radius $R_{\mathrm{c}}$ where the mass expansion becomes sonic, and $\rho_{\mathrm{R}}(R) \propto \exp \left(-M_{\mathrm{a}}^{2} / 2\right)$ [ $M_{\mathrm{a}}=$ Mach number] for $R \gtrsim R_{\mathrm{c}}$ (Arthur et al. 1994; Meilland et al. 2006). These interactions should also produce shock fronts where $T_{\mathrm{e}} \gg T_{\text {eff }}$ (Hartquist et al. 1986; Dyson \& Hartquist 1992; Arthur et al. 1994) where the condition $v_{\mathrm{s}} \ll v_{\phi}$ no longer holds.

2) Magnetic fields of some $10^{2} \mathrm{G}$ have recently been detected in some Be stars and in other active B-type stars (Neiner et al. 2003; Neiner 2006). The decay time of magnetic fields in the highly ionized discrete ejecta and winds is $\tau_{\mathrm{d}} \sim 4 \pi \sigma(l / c)^{2} \sim$ $10^{7} \mathrm{yr}$ (Mestel 2003; $\sigma=$ conductivity; $l \approx 0.1 R_{\mathrm{O}}=$ typical length scale), which is larger by many orders of magnitude than any observed dissipation time of CD in Be stars. The CD material then becomes magnetized and the thin-disc condition used above transforms into $\left(v_{\mathrm{s}}^{2}+2 v_{\mathrm{A}}^{2}\right)^{1 / 2} \ll v_{\mathrm{K}}$, where $v_{\mathrm{A}}$ is the Alfven velocity. For $B \sim 10^{2} \mathrm{G}$ we have $\left(v_{\mathrm{s}}^{2}+2 v_{\mathrm{A}}^{2}\right)^{1 / 2} \sim v_{\mathrm{K}} / 3$ which can again challenge the thin-disc approximation.

\subsection{Enhanced $C D$ scale heights near the star}

We have shown in Sect. 3.1 that under particular physical circumstances, for instance magnetic fields, chromospheric-like temperature structure, discrete mass ejections, etc. the thindisc approximations might not be valid in Be stars. Figure $2 \mathrm{a}$ shows the meridional cuts of isopycnic surfaces of an isothermal Keplerian disc having the density distribution law $N(R) \sim R^{-n(R)}$ given in Sect. 2. We note that the value of $N_{\mathrm{e}}^{\mathrm{o}}$ for this $C D$, deduced with the method described in Sect. 2.3, is still higher than those found for CDs with constant scale heights $h / R_{\mathrm{O}} \lesssim 0.5$. Figure $2 \mathrm{~b}$ shows the cuts of iso-density surfaces of a CD having a chromosphere where $T_{\max }=30000 \mathrm{~K}$ at $R \sim 1.5 R_{\mathrm{o}}$, and the same $N(R)$ as in Fig. 2a. In this case, the enhanced scale height due to the presence of a chromosphere is clearly 


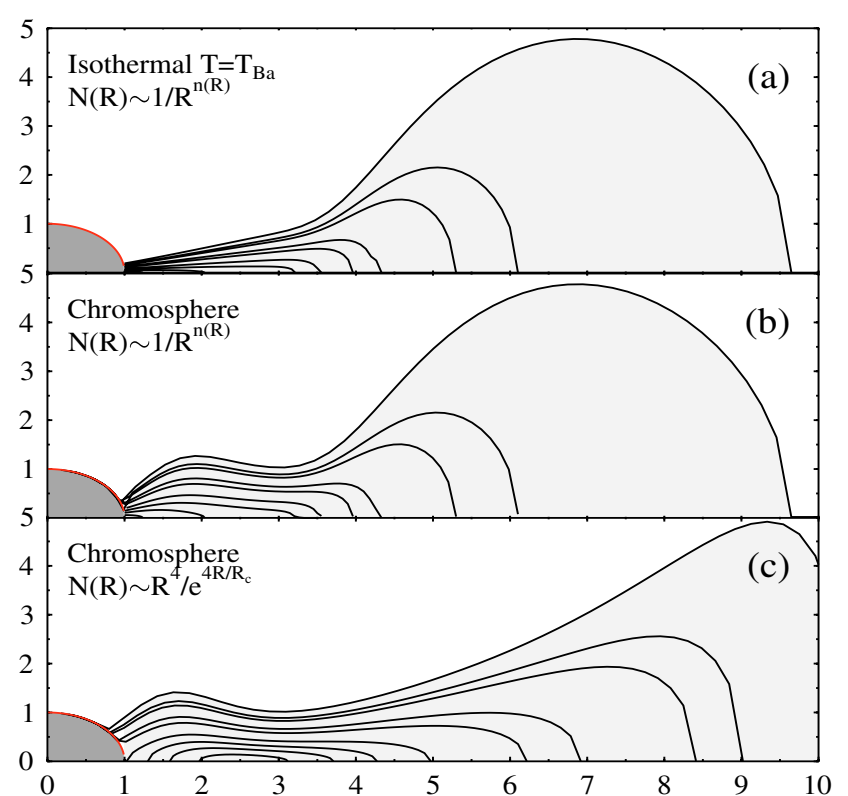

Fig. 2. Shape of discs with Keplerian scale heights. a) Isothermal CD with a density distribution law $N(R) \sim R^{-n(R)}$ where $n(R)=0.5$ for $R<3 R_{\mathrm{o}}$ and $n(R)=3.0$ for $\left.R>3 R_{\mathrm{o}} ; \mathbf{b}\right)$ scale height $H_{z}(R)$ modified by the presence of a chromosphere with $T_{\max }=30000 \mathrm{~K}$ at $R \sim 1.5 R_{\mathrm{o}}$ and the same density structure as in a); b) scale height $H_{z}(R)$ determined by the same chromospheric-like temperature structure as in b) and a density structure given by sketched wind-disc interaction effects.

seen. Figure 2c shows the cuts of isopycnic surfaces obtained with $N(R) \sim R^{m} \exp \left(-m R / R_{\mathrm{c}}\right)\left(m=4\right.$ and $\left.R_{\mathrm{c}}=2.5\right)$, which vaguely mimics the effects due to the wind-disc interaction effects commented in Sect. 3.1. We used in this case the same chromospheric-like temperature structure as for Fig. 2b. We note that the higher densities of the disc in Fig. 2c are at distances $R \sim R_{\mathrm{c}}$, which produces a ring-like structure. All density distributions used in Fig. 2 are exponentials in the $z$-coordinate according to Eq. (4) with scale heights $H_{z}(R)=\left(v_{\mathrm{s}} / v_{\mathrm{K}}\right) R$, where $v_{\mathrm{s}}=(P / \rho)^{1 / 2}$ with $P=P_{\text {gas }}+P_{\text {rad }}$, which varies with $R$ according to the adopted density and temperature structure. In all cases we used the same particle density $N_{\mathrm{o}}=10^{13} \mathrm{~cm}^{-3}$ at $R=R_{\mathrm{o}}$ and the same ratios $10^{-3} \leq N / N_{\mathrm{o}} \leq 1$ to draw the isopycnic curves. Finally, if we convert the particle distribution shown in Fig. 2c into an average density law $N(R) \sim R^{-n}$ valid only for $1 \lesssim R / R_{\mathrm{o}} \lesssim 3$, we obtain $n \lesssim 0.5$.

In what follows, we discuss the incidence of enhanced scale heights on the interpretation of polarimetric, IR photometric, spectroscopic and interferometric data of Be stars.

\section{Incidence of enhanced CD scale heights on observations}

\subsection{Polarimetry}

From a single-scattering analysis, Fox (1993) concluded that the wide and magnetically-supported discs formulated by Ringuelet \& Iglesias (1991) account for the observed range of polarization in Be stars if magnetic fields are $B \lesssim 10^{2} \mathrm{G}$. The density distribution in these discs is described by:

$\rho(R, \theta)=\rho_{\mathrm{R}}(R)\left[1+f(R, n) \sin ^{2} \theta\right]$,

where $\theta$ is the colatitude angle and $f(R, n)$ is a function that depends on the ratio of magnetic to gas pressures and on the induced electric current. This model also predicts that the temperatures required to produce $\mathrm{C}$ IV and Si IV lines may partly be due to dissipation of magnetic energy in disc regions close to the central star.

Disc density distributions similar to the magneticallysupported ones given by (5) have also been studied by Wood et al. $(1996,1997)$ to interpret the spectropolarimetric data of Be stars. These authors replaced $f(R, n)$ by a constant factor $A \sim 10^{3}$ and $\sin ^{2} \theta$ by $\sin ^{m} \theta$. Thick discs are for $m \lesssim 10$, while thin disks are obtained for $m \gtrsim 10^{2}$. Using a multiple photon scattering approach, these authors have shown that isothermal disks with two different opening angles, thus implying geometrically thick and thin $C D$ respectively, can reproduce the visible and IR spectropolarimetry, and the spectrophotometry of the Be star $\zeta$ Tau (Wood et al. 1997). The best fit is nonetheless obtained with a thin disc having an opening angle $\Delta \theta \lesssim 3^{\circ}$, which can also be better supported by the interferometric measurements (Quirrenbach et al. 1997). However, $\zeta$ Tau has a flat and dimpled near-UV spectropolarimetry, which seems difficult to explain (Wood et al. 1997).

To examine the effects on the linear polarization in the visual continuum spectrum produced by discs with scale heights slightly larger than allowed for by the thin Keplerian discs, we have rewritten our multiple photon scattering Monte Carlo calculation code (Zorec et al. 1989; Höflich \& Zorec 1988) so as to make it similar to the one used by Wood et al. $(1996,1997)$. However, contrary to their CD models, our discs are not isothermal, which makes the release of photons in the CD controlled by the local volume recombination $(b f)$ and free-free $(f f)$ emission coefficient $j_{\lambda}$ of hydrogen.

The linear polarization was calculated in the non-LTE approximation. The non-LTE level population deviation coefficients of the Balmer and Paschen hydrogen atomic levels were obtained by detailed calculations of the radiative transfer in spherical envelopes (Cidale \& Ringuelet 1993), for which we have assumed the same radial density and temperature distributions as in the studied CDs. The non-LTE deviation coefficients of the hydrogen Balmer and Paschen levels have a similar behavior to those computed for the average Fe II $m 38$ transition shown in Fig. $1 \mathrm{~b}$, where $b_{\mathrm{u}} / b_{1} \propto R^{2}$ in $R \gtrsim 2.5 R_{\mathrm{o}}$.

Here, we reproduce the polarization results obtained for two test wedge-shaped discs (see Figs. 3a,b), whose geometries were chosen to simplify some calculation steps. While in Keplerian $\mathrm{CD}$ the scale height varies with the distance $R$ to the star as $H_{z}(R)=\left[v_{\mathrm{s}}(R) / \sqrt{G M_{*}}\right] R^{3 / 2}$ and the surface density as $\Sigma_{s}^{K}(R)=$ $\sqrt{\pi} \rho_{\mathrm{R}}(R) H_{z}(R)$, in the wedge-like CD it is $\Sigma_{s}^{W}(R)=\rho_{\mathrm{R}}(R) Z(R)$, where the disc height $Z(R)$ increases linearly with $R$. The heights of wedge-like discs in Figs. 3a,b were adjusted to make their masses integrated up to some $10 R_{\mathrm{O}}$ radii roughly of the same order as of Keplerian discs: $h / R_{\mathrm{O}}=0$ and $h / R_{\mathrm{o}}=0.7$ at $R=R_{\mathrm{o}}$, and $H=5 R_{\mathrm{O}}$ at $R=10 R_{\mathrm{0}}$. For all cases we have adopted the particle density distribution law $N(R) \sim R^{-n(R)}$ with $n(R)$ given in Sect. 2, and $N_{\mathrm{o}}=10^{12} \mathrm{~cm}^{-3}$ at $R=R_{\mathrm{o}}$. The adopted density distribution law is shown in Fig. 3a, where we have also superimposed the extreme distributions determined with constant power indices $n=0.5$ and $n=3.0$. At each radius, the density distribution in the z-coordinate is uniform, as in the wedge-shaped CD models used by Waters (1986). Finally, we note that the wedgeshaped CDs used here do not contradict the results obtained in Sect. 2 for thin CDs with $h / R_{\mathrm{o}}=$ const., because the low value of the source function of Fe II lines beyond $R \gtrsim 3 R_{\mathrm{o}}$ makes the distant and widely "opened" CD regions not contribute to the observed line radiation. 
The residual linear polarization against the wavelength gives, in principle, a mirror image of the $b f+f f$ hydrogen absorption in CDs. This means that the degree of polarization is globally higher in those wavelengths where the $b f+f f$ absorption is smaller, so that the flux of scattered photons can be larger. In general, the degree and wavelength distribution of the polarization is a function of: $i$ ) the electron density, which controls the photon multi-scattering process; $i i)$ the density and temperature distributions in the disc, on which the $b f+f f$ absorption depends and determines the flux of scattered photons; iii) the degree of cancellation of positively polarized photons (photons scattered in the equatorial regions of the $\mathrm{CD}$ ) by photons scattered at higher $\mathrm{CD}$ latitudes, i.e. negatively polarized photons (Wood et al. 1996, 1997).

Since in CD with enhanced disc scale heights near the star the fraction of negatively polarized photons and the $b f+f f$ optical depths can be larger, the expected linear polarization can be lower than the polarization induced by thin discs. Moreover, in our calculations we have used a radius-dependent power index $n(R)$ of the radial particle density distribution and several temperature distributions: isothermal with $T=T_{\mathrm{Ba}}$; radiative equilibrium-like distribution (RE); a chromospheric like temperature distribution (non-RE) followed by an extended isothermal region with $T=T_{\mathrm{Ba}}$. The results thus obtained in the $\lambda \lambda 1500-8000 \AA$ wavelength interval and for an inclination angle $i=86^{\circ}$ are shown in Figs. 3d-f respectively. For comparison, we added a case where discs are isothermal $\left(T=T_{\mathrm{Ba}}\right)$ and have a particle distribution law $N \sim R^{-3}$ (Fig. 3c).

Although the enlarged disc scale heights produce a slightly lower degree of polarization than in thin discs, as expected, the comparison of results shown in Figs. $3 \mathrm{~d}$ to $\mathrm{f}$ with those in Fig. 3c shows that the more striking effect is introduced by the radial particle distribution, represented by a power law where the power index is $n=n(R)$.

As in our simulations $n(R) \sim 0.5$ at $R \lesssim 3 R_{0}$, both the number of photons emitted by the disc and the rate of $b f+f f$ absorptions near the star are higher than in discs having a constant $n \gtrsim 0.5$ over their whole extent. The increase of the $b f+f f$ absorption in the Balmer continuum is then increased, which considerably reduces the number of scattered photons in the nearUV. On the other hand, the ratio $j_{\lambda} / F_{\lambda}$, where $F_{\lambda}$ is the stellar flux, becomes strongly dependent on the disc average radiation temperature, which is lower than the stellar one. The increase of $j_{\lambda}$ induces a larger number of unscattered photons. The strengthened dependence of $j_{\lambda} / F_{\lambda}$ on a low radiation temperature then induces a flat spectral polarization distribution having a different curvature than the polarization produced by discs with a high and constant value of the power index $n$. The combined effect of the increased $b f+f f$ absorptions and emission ratio $j_{\lambda} / F_{\lambda}$ leads to a rather flat near-UV polarization distribution over a fairly large wavelength interval, which might fit the near-UV spectropolarimetry of $\zeta$ Tau, a kind of paradigm of spectropolarimetry in Be-shell stars. Moreover, the dimpled UV polarization of $\zeta$ Tau near $\lambda 2000 \AA$ could be reproduced if the metal-line absorption complexes in the UV are considered, as suggested by Wood et al. (1997). Detailed discussions on the polarization induced by non-isothermal discs with several density distributions and geometries will be presented elsewhere.

\subsection{The IR photometry}

van Kerkwijk et al. (1995) have studied the correlation of the observed $\mathrm{H} \alpha$ emission intensity of Be stars with the near-IR
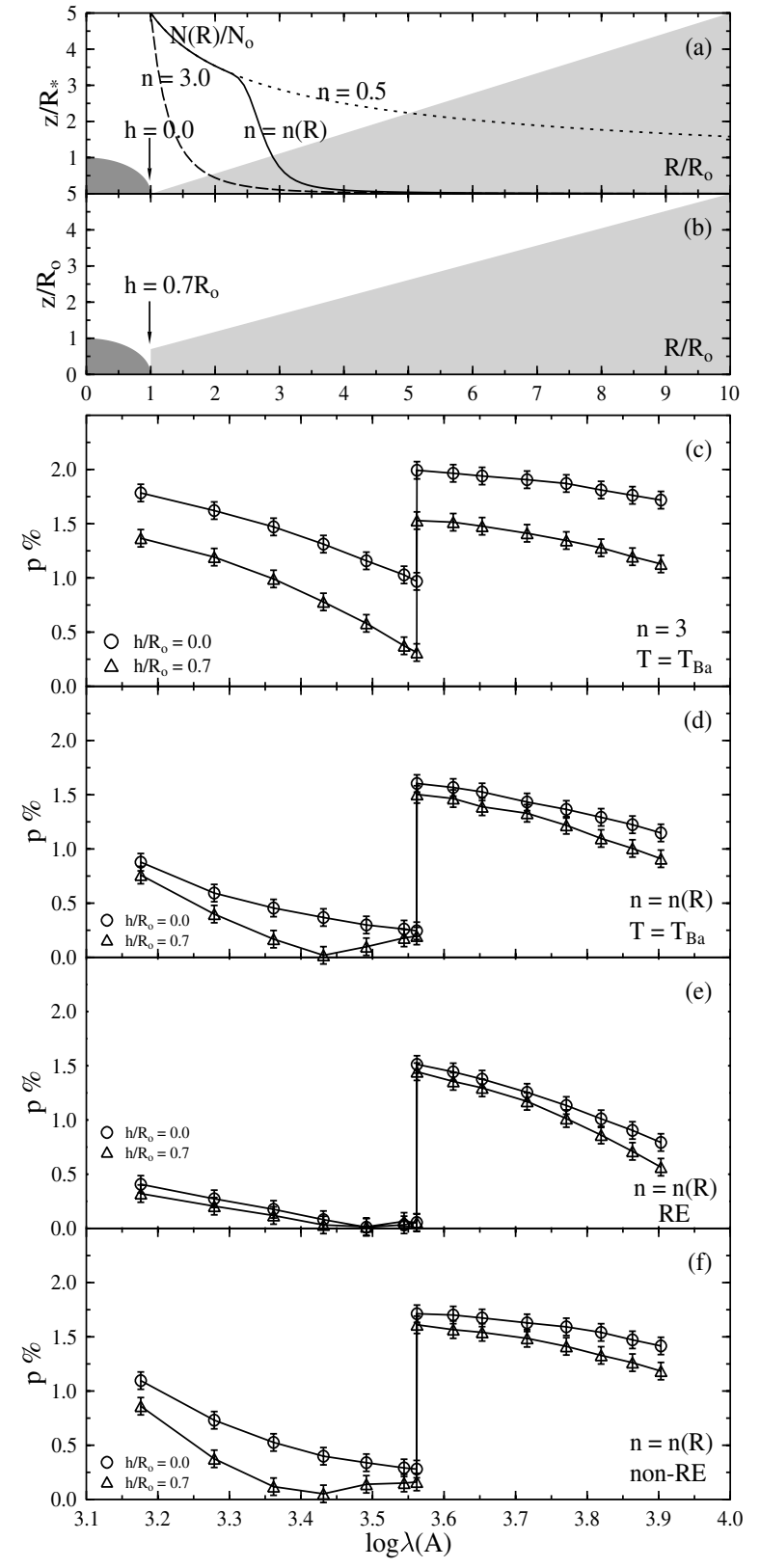

Fig. 3. a) and b) disc models with scale height near the star $h=0$ and $h=0.7 R_{\mathrm{o}}$ used for the polarization calculations. In a) we added the used particle density structure $N(R) / N_{\mathrm{o}}$, which is compared with those with constant values of the power index $n=0.5$ and 3.0. c) residual polarization against the wavelength for isothermal CD and $n=3.0$. d) to f): residual polarization for discs having particle distribution $N(R) / N_{\mathrm{o}}=$ $\left(R_{\mathrm{O}} / R\right)^{n(R)}$ and temperature distributions isothermal, RE and non-RE, respectively. The statistical errors are based on $10^{7}$ incident photons.

$E(J-L)$ color excess. Using isothermal CD models, these authors have shown that: i) the CD model of Poeckert \& Marlborough (1978) (PM), which has an exponential density structure in the $z$-direction, produces an $\mathrm{H} \alpha$ emission which is weaker than that observed for a given $E(J-L)$ color excess; ii) Waters' (1986) CD models (W) produce too strong H $\alpha$ emission for the same $E(J-L)$ color excess. The $\mathrm{W}$ disc models have similar geometrical shapes as those shown in Fig. 3, but $h / R_{\mathrm{o}} \simeq 0$. van Kerkwijk's et al. (1995) calculations show that to explain the observed [H $\alpha, E(J-L)]$ correlation, models must be able to produce intermediate solutions between those obtained with $\mathrm{W}$ 
Table 3. Comparison of masses contained up to radii $R_{\lambda}$ in wedge-like $\mathrm{CD}$ with enhanced scale heights near the star $\left(M_{\mathrm{d}}\left(h, R_{\lambda}\right)\right)$ with those in Keplerian CD $\left(M_{\mathrm{K}}\left(R_{\lambda}\right)\right)$. Estimates of the IR magnitude excesses $\Delta m_{\lambda}$ produced by wedge-like CD with scale heights $h / R_{\mathrm{o}} \neq 0$ near the star as compared to those produced by wedge-like CD with $h / R_{\mathrm{o}}=0$.

\begin{tabular}{c|cccccccccc}
\hline \hline$\lambda \mu \mathrm{m}$ & 1.25 & 2.20 & 3.40 & 5.00 & 10.20 & 12.0 & 25.0 & 60.0 & 100.0 \\
$R_{\lambda} / R_{\mathrm{o}}$ & 1.44 & 2.80 & 3.03 & 3.24 & 4.05 & 4.37 & 5.46 & 7.34 & 7.96 \\
\hline & \multicolumn{10}{c}{$M_{\mathrm{d}}\left(h, R_{\lambda}\right) / M_{\mathrm{K}}\left(R_{\lambda}\right)$} \\
$h / R_{\mathrm{o}}$ & \multicolumn{10}{c}{ Isothermal } \\
0.0 & 0.46 & 1.11 & 1.03 & 0.96 & 0.80 & 0.76 & 0.66 & 0.60 & 0.59 \\
0.5 & 2.18 & 2.06 & 1.88 & 1.74 & 1.44 & 1.36 & 1.18 & 1.01 & 0.99 \\
1.0 & 3.90 & 3.01 & 2.73 & 2.53 & 2.08 & 1.97 & 1.69 & 1.43 & 1.39 \\
\hline$h / R_{\mathrm{o}}$ & \multicolumn{10}{c}{$\mathrm{RE}$} \\
0.0 & 0.35 & 1.44 & 1.39 & 1.35 & 1.22 & 1.12 & 0.92 & 0.77 & 0.75 \\
0.5 & 1.68 & 2.68 & 2.55 & 2.45 & 2.20 & 2.02 & 1.62 & 1.31 & 1.25 \\
1.0 & 3.00 & 3.91 & 3.71 & 3.56 & 3.18 & 2.91 & 2.33 & 1.85 & 1.76 \\
\hline$h / R_{\mathrm{O}}$ & \multicolumn{10}{c}{ non-RE } \\
0.0 & 0.33 & 0.49 & 0.48 & 0.47 & 0.44 & 0.43 & 0.40 & 0.39 & 0.39 \\
0.5 & 1.58 & 0.90 & 0.88 & 0.86 & 0.79 & 0.77 & 0.71 & 0.66 & 0.66 \\
1.0 & 2.83 & 1.32 & 1.29 & 1.25 & 1.14 & 1.10 & 1.02 & 0.94 & 0.92 \\
\hline$h / R_{\mathrm{O}}$ & \multicolumn{10}{c}{$\Delta m_{\lambda} \mathrm{mag}$} \\
0.5 & -1.69 & -0.67 & -0.66 & -0.65 & -0.64 & -0.64 & -0.62 & -0.58 & -0.56 \\
1.0 & -2.33 & -1.08 & -1.06 & -1.05 & -1.04 & -1.03 & -1.01 & -0.95 & -0.93 \\
\hline
\end{tabular}

models for density distributions with $n \sim 3$, and those with the PM models.

Let us briefly comment on the trends of the IR flux excesses produced at $\lambda \gtrsim 1 \mu \mathrm{m}$ by CDs with enhanced scale heights. We first estimate the radii $R_{\lambda}$ of regions up to which $90 \%$ of the emergent flux is produced at a given wavelength $\lambda$, by using non-LTE models of spherical circumstellar envelopes (Cidale \& Vázquez 1993). We then calculate the amount of mass contained up to radii $R_{\lambda}$ in different types of CDs and compared them to each other. In Table 3 we give the formation radii $R_{\lambda}$ calculated for isothermal envelopes having a temperature $T=T_{\mathrm{Ba}}$. In all cases we used the particle density law $N(R) \sim R^{-n(R)}$, where $n=0.5$ at $R \lesssim 3 R_{\mathrm{o}}$ and $n=3.0$ at $R \gtrsim 3 R_{\mathrm{o}}$. Although the radii $R_{\lambda}$ depend slightly on the adopted temperature and density structure of envelopes, the values given in Table 3 are representative of all the studied cases. This table also lists the CD mass ratio "wedge-like/Keplerian": $M_{\mathrm{d}}\left(h, R_{\lambda}\right) / M_{\mathrm{K}}\left(R_{\lambda}\right)$ as a function of the scale height $h / R_{\mathrm{o}}$ and radii $R_{\lambda}$, and for different temperature structures. We can see that in most cases $M_{\mathrm{d}} \gtrsim M_{\mathrm{K}}$, but $M_{\mathrm{d}} / M_{\mathrm{K}} \rightarrow 1$ at large radii $R_{\lambda}$. Thus, the wedge-like CDs have generally more mass than Keplerian discs. The same is also true in the wedge-shaped CD with $h / R_{\mathrm{O}} \neq 0$ as compared to those with $h / R_{\mathrm{O}}=0$. This "mass excess" depends on the temperature structure and on the scale heights near the star. To get a rough estimate of the IR flux excess that wedge-shaped CD with $h / R_{\mathrm{O}} \neq 0$ can produce as compared to wedged CD with $h / R_{\mathrm{O}}=0$, we make the daring assumption that discs are optically thin to the IR radiation, so that the IR flux excesses are simply proportional to the mass contained in the CD (Lamers $\&$ Waters 1984). The IR magnitude excesses are then given by $\Delta m_{\lambda}=-2.5 \log \left[M_{\mathrm{d}}(h \neq 0) / M_{\mathrm{d}}(h=0)\right] \mathrm{mag}$, many of which can be overestimated. These excesses and their distribution will be treated consistently with the polarization in another contribution. However, Table 3 shows the kind of IR flux excesses that can be expected from CDs with $h / R_{\mathrm{o}} \neq 0$.

From Table 3 we see that the radiation in the $J(\lambda \sim 1.25 \mu \mathrm{m})$ and $L(\lambda \sim 3.78 \mu \mathrm{m})$ bands studied by van Kerkwijk et al. (1995) originates in $\mathrm{CD}$ regions with radii $R_{\lambda} \lesssim 3.2 R_{0}$. On the other hand, interferometric estimates of the $\mathrm{H} \alpha$ emission line formation region stretches, according to the star, from $R_{\mathrm{H} \alpha}=7 R_{\mathrm{o}}$ to $32 R_{\mathrm{o}}$ (Tycner et al. 2005), so that $R_{\mathrm{H} \alpha} \gg R_{\text {near-IR }}$. Moreover, it is also known that for effective temperatures of B-type stars, the $\mathrm{H} \alpha$ line source function does not depend on the density of the gas, but on the stellar radiation field (see Sect. 4.3). It is expected then that for the same physical input concerning the $\mathrm{H} \alpha$ source function and for intermediate inclination angles, $C D$ with $h \neq 0$ and $h=0$ would produce about the same $\mathrm{H} \alpha$ luminosity. Nevertheless, discs with $h \neq 0$ can produce larger IR flux excesses than those with $h=0$, so that they may help to account better for the observed $[\mathrm{H} \alpha, E(J-L)]$ correlation.

\subsection{Ho spectroscopy}

In this section we present some arguments based on model spectroscopy which also support enhanced scale heights of $\mathrm{CD}$ in $\mathrm{Be}$ stars near the central object.

Quantitative analysis of Balmer line emission intensities shows that isothermal Keplerian disc models need electron densities $N_{\mathrm{e}} \approx 10^{14} \mathrm{~cm}^{-3}$ at $R=R_{\mathrm{o}}$ to produce sufficiently strong $\mathrm{H} \alpha$ emission (Hummel 1994). However, these densities are comparable to that of photospheric layers producing $\mathrm{H} \alpha$ absorption. The necessity for such high densities is due to the assumed distribution $N(R) \propto R^{-n}$ with $n=3$ which implies a steep CD density decrease with distance. In addition, these models have a collision-dominated $\mathrm{H} \alpha$ source function, which mirrors the disc density distribution and thus misleadingly overpowers the CD emission. It is noteworthy that even the two-level atom approximation with transitions to the continuum is able to show that the $\mathrm{H} \alpha$ source function has several terms. Their importance varies according to the physical circumstances and change its nature and its emitting power (Mihalas 1978):

$S_{\mathrm{H} \alpha}=\left[\int_{0}^{\infty} \phi_{\mathrm{H} \alpha} J_{\lambda} \mathrm{d} \lambda+\epsilon B_{\mathrm{H} \alpha}\left(T_{\mathrm{e}}\right)+\eta B_{\mathrm{H} \alpha}^{*}\right] /[1+\epsilon+\eta]$,

where the first term accounts for the $\mathrm{H} \alpha$ radiation field; $\epsilon B\left(T_{\mathrm{e}}\right)$ and $\eta B_{\mathrm{H} \alpha}^{*}$ are the collision and radiative "source" terms, respectively; $\epsilon$ and $\eta$ are the thermal and radiative "sink" terms, respectively (see Mihalas 1978, p. 358 for detailed description of (6)). To identify the actual nature of $S_{\mathrm{H} \alpha}$ in Be stars, we calculated these terms for the following average physical conditions: $N_{\mathrm{e}} \lesssim 10^{13} \mathrm{~cm}^{-3}, T_{\mathrm{e}} \sim 10^{4} \mathrm{~K}$ (Waters \& Marlborough 1992) and effective temperatures $10^{4} \lesssim T_{\text {eff }} \lesssim 35000 \mathrm{~K}$. We thus obtain:

$$
\left.\begin{array}{c}
0.01 \lesssim \epsilon B\left(T_{\mathrm{e}}\right)_{\mathrm{H} \alpha} / \eta B_{\mathrm{H} \alpha}^{*} \\
0.01 \lesssim \epsilon / \eta \lesssim 0.2
\end{array}\right\}
$$

which implies that in CD of Be stars the $S_{\mathrm{H} \alpha}$ is photoionizationdominated (Ph-D) at more than $90 \%$ on average. $S_{\mathrm{H} \alpha}$ is then almost insensitive to the electron temperature distribution in the disc and it cannot mirror its isopycnic contours. Instead, the PhD source function decreases with the distance from the star and rapidly becomes ineffective at producing strong emission. This decrease can be proved by noting that $S_{\mathrm{H} \alpha} \propto\left(b_{3} / b_{2}\right)\left(N_{3}^{*} / N_{2}^{*}\right)$ $\left(N_{n}^{*}=\right.$ LTE atomic level populations). In our non-LTE models the $\mathrm{H}$ deviation coefficients for Balmer and Paschen levels behave in a similar way as those depicted in Fig. 1b. In particular $b_{3} / b_{2} \propto R^{-2}$ at $R \gtrsim 2$, so that also $S_{\mathrm{H} \alpha} \propto R^{-2}$. The Be star line emission can then be raised to suitable intensity levels with reasonable disc densities $\left(N_{\mathrm{e}} \lesssim 10^{13} \mathrm{~cm}^{-3}\right)$ if it is $N(R) \propto R^{-n}$ with $n<3$ near the star and if the emitting surface there is larger than allowed for by RE Keplerian discs (Levenhagen et al. 2003; 
Arias 2004; Arias et al. 2007; Vinicius et al. 2006). This is particularly significant for many Be stars with high $V \sin i$ values and strong $\mathrm{H} \alpha$ emission which must be produced by a $\mathrm{CD}$ seen equator-on.

\subsection{Interferometry}

The recent interferometric determinations of apparent CD axial ratios $\mathcal{R}(i)=R_{\text {pole }}(i) / R_{\text {equat }}$ are considered as strong observational evidence that Be star discs are geometrically thin (Quirrenbach et al. 1997; Tycner et al. 2005, 2006). For a thin disk it holds then that $\mathcal{R}_{\text {thin }}\left(i_{\text {thin }}\right)=\cos i_{\text {thin }}$ where $i_{\text {thin }}$ is the aspect angle. However, if the CD had an oblate structure with an effective semi-height $H_{\text {pole }}$ at $R \approx R_{\mathrm{o}}$, the axial ratio $\mathcal{R}=H_{\text {pole }} / R_{\text {eq }}$ would imply that the observed, aspect angle-dependent axial ratio is $\mathcal{R}_{\text {thick }}=\left[1-\left(1-\mathcal{R}^{2}\right) \sin ^{2} i_{\text {thick }}\right]^{1 / 2}$. So, the inclination $i_{\text {thin }}$ associated with a thin disc representation might mask an inner CD structure that is slightly thicker, with $0 \leq \mathcal{R} \leq \mathcal{R}_{\text {thin }}$ in which case the inclination angle of the CD is $i_{\text {thick }} \geq i_{\text {thin }}$. We note that the smallest $\mathrm{CD}$ axial ratios observed up to now are for $\zeta$ Tau and $\Phi$ Per, where $\mathcal{R}(i) \simeq 0.3$ (Quirrenbach et al. 1997; Tycner et al. 2006). Assuming that the inclination angle of CD in $\zeta$ Tau is the value inferred by Wood et al. (1997) with their thin-disc solution: $i \simeq 82^{\circ}$, we deduce $\mathcal{R}=0.27$. Tycner et al. (2005) obtained that the radius of the $\mathrm{H} \alpha$ emitting region in the $\mathrm{CD}$ radius of $\zeta$ Tau is $R_{\text {eq }} \simeq 7.2 R_{\mathrm{o}}$ at an epoch when $\mathcal{R}(i) \simeq 0.3$. It follows then that the scale height of the CE near central star can be as high as $H_{\text {pole }} \simeq 2 R_{0}$, which is still consistent with the existent interferometric measurements of $\zeta$ Tau. The same calculation for $\Phi$ Per with data from Tycner et al. $(2005,2006)$ leads to $H_{\text {pole }} \simeq 1.2 R_{\mathrm{o}}$.

\section{Discussion and conclusions}

Using simple CD models, we inferred the density distribution and the scale height of $\mathrm{CD}$ regions where the Fe II emission lines of Be stars are formed. We have done this by accounting for the average pole-on and equator-on optical depths of these lines determined with the SAC method. Calculations were performed through a non-LTE approximation of the average Fe II level populations of multiplet 38 . We concluded that the Fe II emission line formation region should have the following characteristics: a) not to extend farther out than some $R \sim 3 R_{\mathrm{O}}$ ( $R_{\mathrm{o}}=$ stellar radius $\left.) ; \mathrm{b}\right)$ its $z$-scale height near the star must be $h / R_{\mathrm{O}} \gtrsim 0.5$; c) the power index of the particle density distribution $N(R)=N_{\mathrm{o}}\left(R_{\mathrm{o}} / R\right)^{n}$ in $R \lesssim 3 R_{\mathrm{o}}$ must be $n \lesssim 1$. Since in regions where the far-IR flux excesses of Be stars are formed, the power index of the density distribution must be $2 \lessgtr n \lesssim 3.5$, we concluded that the particle density distribution in $\mathrm{CD}$ of $\mathrm{Be}$ stars has a power index $n(R)$ that increses with $R$.

Larger scale heights near the star than in isothermal Keplerian thin discs can be justified either by the presence of wind-disc interaction effects and non-RE temperature distributions, and/or by magnetic fields $B \sim 10^{2} \mathrm{G}$.

Photon multi-sacttering Monte Carlo simulations of the linear polarization induced by discs with enhanced scale heights near the star show that polarimetric measurements cannot exclude the existence of enhanced scale heights of $\mathrm{CD}$ in regions close to the central star. Moreover, the non-LTE simulations of the linear polarization produced in CDs with particle density distributions $N \sim R^{-n}$, where $n$ is a function of $R$, can explain the presence of flat polarization distributions in the near-UV, nearly as observed in $\zeta$ Tau.

We argued that the CDs with enhanced scale heights near the star can produce about the same $\mathrm{H} \alpha$ line emission, but larger IR flux excesses than thin discs. These CD could then explain the observed [H $\alpha, E(J-L)]$ correlation.

We have noted that consistent radiative transfer calculation of Balmer emission lines requires larger effective emitting surfaces than allowed for by RE Keplerian CD. We concluded that the enhanced disc scale heights near the star do not contradict the existent interferometric measurements of Be star discs.

Finally, we hazard the speculation that the inferred enhanced disc scale heights could mask some possible transition region from the hot polar plumes to the equator, recently suggested by Kervella \& Domiciano (2006). We shall test elsewhere whether these regions can produce spectroscopic and polarimetric effects similar to those described in this paper. To this end, detailed analysis of expanding velocities and modeling of Fe II emission line profiles in Be stars are in progress.

Acknowledgements. J.Z. thanks Drs. A. Domiciano de Souza, O. Chesneau, P. Stee and R. Rohrmann for discussions on the interferometry and physics of discs in Be stars. We are greatly indebted to an anonymous referee for criticisms and suggestions that helped to improve this work substantially. We thank M. Usdin for the language editing of this paper. L.C. acknowledges financial support from the Agencia de Promoción Científica y Tecnológica (BID 1728 OC/AR PICT 03-12720) and the Programa de Incentivos G11/073 of the National University of La Plata.

\section{References}

Arias, M. L. 2004, Ph.D. Thesis, University of La Plata, Argentina Arias, M. L, Zorec, J., Cidale, L., et al. 2006, A\&A, 460, 821

Arias, M. L., Zorec, J., Frémat, Y. 2007, ASP Conf. Ser., 361, 419 Apparao, K. M. V., Antia, H. M., \& Chitre, S. M. 1987, A\&A, 177, 198

Arthur, S. J., Dyson, J. E., \& Hartquist, T. W. 1994, MNRAS, 269, 1117

Brown, J. C., \& Wood, K. 1992, A\&A, 265, 663

Carciofi, A. C., \& Bjorkman, J. E. 2006, ApJ, 639, 1081

Carciofi, A. C., Miroshnichenko, A. S., Kusakin, A. V. et al. 2006, ApJ, 652, 1617

Cidale, L. S. 1998, ApJ, 502, 824

Cidale, L. S., \& Ringuelet, A. E. 1989, PASP 101, 417

Cidale, L. S., \& Ringuelet, A. E. 1993, ApJ, 411, 874

Cidale, L. S., \& Vázquez, A. C. 1995, ApJ, 453, 393

Cidale, L. S., Zorec, J. Maillard, J. P., \& Morrell, N. 2000, IAU Coll., 175, 472

Dyson, J. E., \& Hartquist, T. W. 1992, Astro. Lett. Comm., 28, 301

Floquet, M., Hubert, A. M., Hirata, R., et al. 2000, A\&A, 362, 1020

Fontenla, J. M., Rovira, M., \& Ringuelet, A. E. 1981, Rev. Mex. Astron. Astrofis., 6, 209

Fox, G. K. 1993, MNRAS, 260, 525

Frank, J., King, A., \& Raine, D. 1992, Accretion Power in Astrophysics, 2nd edn., Cambridge Astroph. Series

Frémat, Y., Zorec, J., Hubert, A. M., et al. 2005, A\&A, 440, 305

Friedjung, M., \& Muratorio, G. 1987, A\&A 188, 100

Guinan, E. F., \& Hayes, D. P. 1984, ApJ., 287, L39

Hanuschik, R. W., Dachs, J. Baudzus, M., \& Thimm, G. 1993, A\&A, 274, 356 Hartquist, T. W., Dyson, J. E., Pettini, M., \& Smith, L. J. 1986, MNRAS, 221, 715

Höflich, P., \& Zorec, J. 1988, In: Modeling the stellar environment, how and why?, 4th IAP Ap. Meeting (ed. Frontières), 257

Hubert, A. M., Floquet, M., \& Zorec, J. 2000, IAU Coll., 175, 348

Hummel, W. 1994, A\&A, 289, 458

Jefferies, J. T. 1968, Spectral Line Formation (Waltham, MA: Blaisdel)

Kastner, S. O. 1999, A\&A, 351, 1016

Kervella, P., \& Domiciano de Souza, A. 2006, A\&A, 453, 1059

Lamers, H. J. G. L. M., \& Snow, T. P. 1978, ApJ., 219, 504

Lamers, H. J. G. L. M., \& Waters, L. B. F. M. 1984, A\&A, 136, 37

Levenhagen, R. S., Leister, N. V., \& Zorec, J., et al. 2003, A\&A, 400, 599

Meilland, A., Stee, Ph., Zorec, J., \& Kanaan, S. 2006, A\&A, 455, 953

Mestel, L. 2003, Stellar Magnetism, Clarendon Press

Mihalas D. 1978, Stellar Atmospheres, 2nd. ed. (Freeman \& Co.)

Moujtahid A., Zorec, J., \& Hubert A. M. 1999, A\&A, 349, 151

Muratorio, G., \& Friedjung, M. 1988, A\&A 190, 103

Nahar, S. 1995, A\&A, 293, 967

Neiner, C. 2007, ASP Conf. Ser., 361, 91

Neiner, C., Hubert, A. M., Frémat, Y., et al. 2003c, A\&A, 409, 275

Poeckert, R., \& Marlborough, J. M. 1978, ApJ, 220, 940

Quirrenbach, A., Bjorkman, K. S., \& Bjorkman, J. E., et al. 1997, ApJ, 479, 477 
Rohrmann, R. D. 1997, MNRAS, 285, 427

Rohrmann, R. D., Zorec, J., Cidale, L., Morrell, N., \& Frémat, Y. 2003, IAU Symp., 210, A26

Schmitz, F. 1983, A\&A, 120, 234

Snow, T. P. 1981, ApJ, 251, 139

Ringuelet, A. E., \& Iglesias, M. E. 1991, ApJ, 369, 463

Rivinius, T., Baade, D. Štefl, S., et al. 1998, A\&A, 336, 177

Tycner, C., Lester, J. B., Hajian, A. R., et al. 2005, ApJ, 624, 359

Tycner, C., Gilbreath, G. C., \& Zavala, R. T., et al. 2006, AJ, 131, 2710

van Kerkwijk, M. H., Waters, L. B. F. M., \& Marlborough, J. M. 1995, A\&A, 300, 259

Vinicius, M. M. F., Zorec, J., Leister, N. V., et al. 2006, A\&A, 446, 643
Waters, L. B. F. M. 1986, A\&A, 162, 121

Waters, L. B. F. M., Coté, J., \& Lamers, H. 1987, A\&A, 185, 206

Waters, L. B. F. M., \& Marlborough, J. M. 1992, A\&A, 256, 195

Waters, L. B. F. M., Marlborough, J. M., Van der Veen, W.E.C., Taylor, A.R., \& Dougherty, S. M. 1991, A\&A, 244, 120

Wood, K., Bjorkman, J. E., Whitney, B. A., et al. 1996, ApJ, 461, 847

Wood, K., Bjorkman, K. S., \& Bjorkman, J. E. 1997, ApJ, 477, 926

Zhang, H. L., \& Prahdhan, A. K. 1995, A\&A, 293, 953

Zorec, J., \& Höflich, P. 1989, A\&A, 210, 279

Zorec, J., \& Briot, D. 1997, A\&A, 318, 443

Zorec, J., Frémat, Y., \& Hubert, A. M. 2000, IAU Coll., 175, 330

Zorec, J., Frémat, Y., \& Cidale, L. 2005, A\&A, 441, 235 